\title{
Validation of Genome-Wide Association Studies as a Tool to Identify Virulence Factors in Parastagonospora nodorum
}

\author{
Yuanyuan Gao, Zhaohui Liu, Justin D. Faris, Jonathan Richards, Robert S. Brueggeman, Xuehui Li, Richard P. Oliver, \\ Bruce A. McDonald, and Timothy L. Friesen
}

First, second, fourth, fifth, and ninth authors: Department of Plant Pathology, North Dakota State University, Fargo 58108; third and ninth authors: United States Department of Agriculture-Agricultural Research Service, Northern Crop Science Laboratory, Cereal Crops Research Unit, Fargo, ND, 58102; sixth author: Department of Plant Science, North Dakota State University, Fargo; seventh author: Centre for Crop and Disease Management, Department of Environment and Agriculture, School of Science, Curtin University, Perth, WA 6102, Australia; and eighth author: Institute of Integrative Biology, Plant Pathology Group, Swiss Federal Institute of Technology, ETH Zentrum, LFW, CH-8092 Zürich, Switzerland.

Accepted for publication 8 July 2016.

\begin{abstract}
Gao, Y., Liu, Z., Faris, J. D., Richards, J., Brueggeman, R. S., Li, X., Oliver, R. P., McDonald, B. A., and Friesen, T. L. 2016. Validation of genome-wide association studies as a tool to identify virulence factors in Parastagonospora nodorum. Phytopathology 106:1177-1185.

Parastagonospora nodorum is a necrotrophic fungal pathogen causing Septoria nodorum blotch on wheat. We have identified nine necrotrophic effector-host dominant sensitivity gene interactions, and we have cloned three of the necrotrophic effector genes, including SnToxA, SnToxl, and $S n T o x 3$. Because sexual populations of $P$. nodorum are difficult to develop under lab conditions, genome-wide association study (GWAS) is the best population genomic approach to identify genomic regions associated with traits using natural populations. In this article, we used a global collection of 191 P. nodorum isolates from which we identified 2,983 singlenucleotide polymorphism (SNP) markers and gene markers for SnToxA and SnTox3 to evaluate the power of GWAS on two popular wheat breeding lines that were sensitive to SnToxA and SnTox3. Strong marker trait associations (MTA) with P. nodorum virulence that mapped to SnTox3

and SnToxA were first identified using the marker set described above. A novel locus in the $P$. nodorum genome associated with virulence was also identified as a result of this analysis. To evaluate whether a sufficient level of marker saturation was available, we designed a set of primers every $1 \mathrm{~kb}$ in the genomic regions containing SnToxA and SnTox3. Polymerase chain reaction amplification was performed across the 191 isolates and the presence/absence polymorphism was scored and used as the genotype. The marker proximity necessary to identify MTA flanking SnToxA and SnTox3 ranged from 4 to 5 and 1 to $7 \mathrm{~kb}$, respectively. Similar analysis was performed on the novel locus. Using a $45 \%$ missing data threshold, two more SNP were identified spanning a $4.6-\mathrm{kb}$ genomic region at the novel locus. These results showed that the rate of linkage disequilibrium (LD) decay in $P$. nodorum and, likely, other fungi is high compared with plants and animals. The fast LD decay in $P$. nodorum is an advantage only if sufficient marker density is attained. Based on our results with the SnToxA and $S n T o x 3$ regions, markers are needed every 9 or $8 \mathrm{~kb}$, respectively, or in every gene, to guarantee that genes associated with a quantitative trait such as virulence are not missed.
\end{abstract}

Genome-wide association studies (GWAS) provide a powerful approach to analyze DNA sequence variation across a genome with the aim of identifying genetic variation associated with traits of interest in a natural population (Gibson and Muse 2009). Numerous quantitative genetic studies have been used to map quantitative trait loci (QTL) in plants (Zhao et al. 2014). QTL analysis uses designed, biparental populations that segregate for a certain trait or traits. Because GWAS uses naturally diverse populations that maintain a high frequency of recombination events over their evolutionary history, GWAS often has advantages over biparental populations in targeting complex traits because of its relatively high mapping resolution (Doerge 2002; Holland 2007).

Due to the falling cost of next-generation sequencing and other genomic technologies such as high-throughput genotyping, transcriptomics, and comparative genomics (Zhu et al. 2008), GWAS is widely used to identify gene loci for human diseases (Montgomery

Corresponding author: T. L. Friesen; E-mail address: Timothy.Friesen@ars.usda.gov

*The $e$-Xtra logo stands for "electronic extra" and indicates that four supplementary figures and four supplementary tables are published online.

http://dx.doi.org/10.1094/PHYTO-02-16-0113-FI

This article is in the public domain and not copyrightable. It may be freely reprinted with customary crediting of the source. The American Phytopathological Society, 2016.
2011) and plants (Zhu et al. 2008). GWAS has already been applied in maize, Arabidopsis, sorghum, wheat, barley, potato, and rice to genetically characterize complex traits such as flowering time, kernel composition, disease resistance, shoot branching, kernel size, and milling quality (Zhu et al. 2008).

In fungi, only a few studies using GWAS analysis have been reported. In Saccharomyces cerevisiae, a single-nucleotide polymorphism (SNP) associated with mtDNA copy number was identified by GWAS (Connelly and Akey 2012). Muller et al. (2011) analyzed a geographically diverse set of 44 clinical and 44 nonclinical $S$. cerevisiae strains to identify genetic loci associated with clinically relevant traits, including pseudohyphal formation, cell wall maintenance, and cellular detoxification associated with pathogenesis and virulence. Dalman et al. (2013) sequenced 23 haploid isolates of Heterobasidion annosum, a necrotrophic pathogen of Picea abies and Pinus sylvestris, to identify 33,018 SNP. In total, 12 SNP were associated with virulence using GWAS. Genomic regions associated with the 12 SNP contained eight candidate virulence genes with homology to genes in other fungal pathogens. Palma-Guerrero et al. (2013) used GWAS to identify a gene homolog encoding a neuronal calcium sensor associated with germling communication in Neurospora crassa. Talas et al. (2016) genotyped 220 German Fusarium graminearum isolates and identified 50,29 , and 74 quantitative trait nucleotides associated with aggressiveness, deoxynivalenol production, and propiconazole sensitivity, 
respectively. Although GWAS research in fungi remains rare compared with plants and animals, GWAS has significant potential applications in fungi because they have small genomes, often exhibit a haploid chromosomal stage, and often undergo regular sexual cycles that reduce linkage disequilibrium (LD) and result in high levels of diversity among individuals drawn from natural populations (Dalman et al. 2013).

Parastagonospora nodorum produces necrotrophic effectors (NE) that are critical to the virulence of the pathogen. The NE produced by $P$. nodorum are recognized directly or indirectly by dominant wheat sensitivity genes that confer susceptibility. To date, we have cloned three NE genes, including SnToxA, SnToxl, and SnTox3 (Friesen et al. 2006; Liu et al. 2009, 2012). According to previous studies for SnToxA and SnTox3 presence or absence variation across seven global geographic regions (McDonald et al. 2013), the absence of SnToxA ranged from 3\% (Australia) to $94 \%$ (China) and SnTox3 ranged from 4\% (Australia) to 60\% (China). Bertucci et al. (2014) tested 33 P. nodorum isolates collected from the southeastern United States on 25 winter wheat cultivars, from which 25 isolates were used in this study (Supplementary Table S2). Because sexual populations of $P$. nodorum are difficult to produce under lab conditions, biparental populations needed for QTL mapping have not been used to genetically locate genes involved in virulence; namely, genes involved in NE production. An annotated reference genome sequence along with GWAS would provide an alternative way to target complex traits because the population used in GWAS contains natural genetic diversity, with high-frequency evolutionary and historical recombination.

Here, we report on a large set of SNP markers distributed across the $P$. nodorum genome that can be used in a GWAS analysis to identify genomic regions harboring virulence factors important in Septoria nodorum blotch (SNB). We first coupled an SNP marker set with an additional set of markers specifically targeting SnToxA and SnTox3 to show that GWAS could identify marker trait associations (MTA) involving SnToxA and SnTox3. This analysis allowed us to determine the marker density needed to identify significant MTA in these regions on wheat lines known to harbor the corresponding NE sensitivity genes Tsnl (Faris et al. 2010) and Snn3-B1 (Shi et al. 2016). Then, we used the SNP data in a GWAS analysis that led to the discovery of a novel virulence locus affecting the same wheat lines that harbored sensitivity to SnToxA or SnTox3, illustrating the potential of GWAS to identify novel determinants of virulence.

\section{MATERIALS AND METHODS}

Plant and fungal materials. The susceptible wheat 'Sumai3', which contains the NE sensitivity genes Tsn1 and Snn3-B1, was used as a positive control for the SnToxA and SnTox3 genes. The North Dakota hard red spring wheat 'Alsen' (Tsnl) was used as a positive control for the SnToxA as well as to identify a novel locus associated with $P$. nodorum virulence. Both wheat lines were inoculated with spores obtained from a global sample of 191 $P$. nodorum isolates. The 191 P. nodorum isolates originated from 13 U.S. states ( $n=94$ isolates), Australia $(n=30)$, Denmark $(n=2)$, Finland $(n=3)$, Lithuania $(n=1)$, Norway $(n=8)$, Sweden $(n=3)$, Brazil $(n=3)$, Switzerland $(n=10)$, China $(n=18)$, South Africa $(n=5)$, and Iran $(n=14)$ (Supplementary Table S1).

Phenotypic evaluation. The $191 P$. nodorum isolates were grown on V8-potato dextrose agar for 7 days, as described by Friesen and Faris (2010). Conidia (pycnidiospores) served as inoculum and were concentrated to $1 \times 10^{6}$ spores $/ \mathrm{ml}$ and sprayed onto seedlings at the two- to three-leaf stage (Liu et al. 2004b). The inoculated plants were placed at $100 \%$ relative humidity in the light for $24 \mathrm{~h}$ in a mist chamber, then moved to a growth chamber at $21^{\circ} \mathrm{C}$ with a 12 -h photoperiod for six additional days. The disease on the second leaf of each inoculated plant was scored 7 days postinoculation using a 0 -to-5 rating scale based on lesion type, where $0=$ no visible lesions, $1=$ few penetration points with lesions consisting of flecking or small dark spots, 2 = lesions consisting of dark spots with little surrounding necrosis or chlorosis, $3=$ dark lesions with size of 2 to $3 \mathrm{~mm}$ completely surrounded by necrosis or chlorosis, $4=$ larger necrotic or chlorosis $4 \mathrm{~mm}$ or greater with little coalescence, and $5=$ large coalescent lesions with very little green tissue remaining (Liu et al. 2004a). Three replicates were completed for each isolate-wheat line combination, with each replicate consisting of a single cone-tainer containing three seedlings that were evaluated collectively. After identifying SNP associated with a novel virulence identified through the GWAS (as described below in the "LD analysis and the initial association analysis" section), disease reactions of pools of isolates harboring alternative SNP alleles were investigated using a two-tailed $t$ test in Excel to verify the significance of associations between genotypes and phenotypes using a $0.001 P$ value cutoff.

DNA extraction, library preparation, and genotyping. DNA of the 191 P. nodorum isolates was extracted using a BioSprint 15 system (Qiagen). DNA of each isolate was initially genotyped using three methods. The first method used a restriction site associated DNA genotype by sequencing approach (Baird et al. 2008; Elshire et al. 2011) to identify SNP. A total of $60 \mathrm{ng}$ of DNA from each isolate was digested with ApeKI and $H h a \mathrm{I}$ (NEB), then ligated with specific barcoded adaptors, as described by Leboldus et al. (2015). The barcoded DNA of 40 isolates were pooled into one tube, and five pooled genomic DNA libraries were made. A 3- $\mu$ l sample from each pooled library was subjected to electrophoresis on a $1 \%$ agarose gel at $100 \mathrm{~V}$ for $45 \mathrm{~min}$. The DNA fragments between 200 and $250 \mathrm{bp}$ were collected and purified using a gel extraction kit (Qiagen) followed by an additional size selection on a Pippin DNA size selection system (Sage Science) using the Pippin Prep cassette and Pippin Prep reagent kit (Sage Science). Library sequencing was performed using five Ion Torrent 318 microprocessor chips (Life Technologies), as described by Leboldus et al. (2015).

Second, the SnToxA and SnTox3 genes were polymerase chain reaction (PCR) amplified from all 191 isolates using primer sets specific to SnToxA (Friesen et al. 2006) and SnTox3 (Liu et al. 2009) to identify presence/absence variation in these two genes. Primers specific to these two genes can be found in Supplementary Table S1. These genes were used as positive controls to validate the phenotyping and effectiveness of GWAS analysis in P. nodorum.

Additionally, primers were designed to amplify 1-kb DNA fragments along the $20-\mathrm{kb}$ genomic regions containing the SnToxA and SnTox3 genes, and a novel virulence locus identified using the RAD-GBS markers described above. All PCR amplification followed the parameters of $94^{\circ} \mathrm{C} 4 \mathrm{~min} ; 30$ cycles of $94^{\circ} \mathrm{C}$ for $30 \mathrm{~s}$, $60^{\circ} \mathrm{C}$ for $30 \mathrm{~s}$, and $72^{\circ} \mathrm{C}$ for 45 to $90 \mathrm{~s}$ according to the length of the target fragment; followed by $72^{\circ} \mathrm{C}$ for $10 \mathrm{~min}$. The presence or absence markers in the 20-kb genomic regions containing SnToxA, SnTox3, and the novel locus were included in GWAS analysis.

Genotypic data analysis. The $P$. nodorum isolate that had the most reads was de novo assembled as the reference sequence using CLC Genomics Workbench (Qiagen). All the sequencing reads were aligned to the reference sequence using the BurrowsWheeler Aligner 'mem' algorithm (Li and Durbin 2010). Sequence Alignment/Map (SAM) files generated by the Burrows-Wheeler Aligner were converted to Binary Alignment/Map files and subsequently used to identify SNP markers using SAM tools with default settings (Li and Durbin 2010). SNP with low quality $(<100)$ were filtered from the dataset. SNP having a minor allele frequency less than $5 \%$ or missing data greater than $30 \%$ were eliminated. Presence/absence polymorphisms of SnToxA and SnTox3 were identified by PCR. Isolates with positive amplification of SnToxA or SnTox3 were scored as 0 , and isolates without amplification were scored as 1 . When evaluating the novel virulence locus associated with both Alsen and Sumai3, a $45 \%$ missing data cutoff was used to increase the number of markers in that region alone. Individual genotypes with quality $>10$ and minimum read depth of 3 were used. For each sequence tag, the SNP markers with the least 
missing data were chosen, resulting in one SNP marker per tag being used as a genotypic marker. The chosen SNP were used for population structure analysis, relatedness analysis, and association mapping. SNP marker data were imported into Excel and annotated onto the sequence of the $P$. nodorum genome reference strain SN15 (Hane et al. 2007; Syme et al. 2013, 2016).

Population structure and relatedness analysis. The software STRUCTURE 2.3.4 (Pritchard et al. 2000) was used to analyze the population structure of the $191 P$. nodorum isolates using an admixture model with a burn-in period of 10,000 followed by 50,000 Markov Chain Monte Carlo replications. Five runs for each cycle were performed with the cluster number between 1 and 20 . The optimal value of the number of clusters $(k)$ was decided based on the relative rate of change in the likelihood of the value $(\Delta k)$ between two successive clusters. The value of $\Delta k$ was calculated as described by Evanno et al. (2005) using STRUCTURE Harvester (Earl and vonHoldt 2012). Further analyses were performed from $k=2$ to the optimal $k+3$, with a burn-in period of 20,000 followed by 200,000 Markov Chain Monte Carlo replications with 20 iterations. The membership probability of each isolate at the optimal $k$ was used as the Q matrix for further analysis.

Relative kinship was calculated by estimating the pairwise relatedness of the 191 isolates for the 2,983 SNP markers using JMP genomics 6.1. The probability of any two individuals sharing the same allele was computed using Gower's Similarity Metric and a range standardization that is referred to as identity by state (IBS) across all markers (Stevens et al. 2011).

LD analysis and the initial association analysis. LD between SNP markers was estimated as the squared allele frequency correlation $\left(r^{2}\right)$ and calculated using JMP Genomics 6.1. Decay of LD was calculated by fitting an equation developed by Hill and Robertson (1968) into a nonlinear regression curve using JMP Genomics 6.1. The marker saturation necessary to identify MTA was based on the average distance of paired SNP markers at LD level of $r^{2}$ value $\geq 0.8$ (Bastien et al. 2014) and the LD decay at $r^{2} \leq$ 0.1 (Nordborg and Tavare 2002; Palaisa et al. 2004; Remington et al. 2001). Both LD and LD decay $r^{2}$ thresholds indicate a pair of markers are linked. Association analysis was performed in JMP Genomics 6.1 using SNP and SnToxA and SnTox3 gene markers. The SNP with a $-\log _{10}(P$ value $)$ or adjusted $-\log _{10}(P$ value $)$ greater than $4.5(P<0.0000316)$ were considered significant. The naive model (Pasam et al. 2012) and three mixed models, including population structure (Q model), kinship (IBS model) and population structure + kinship (Q+IBS model) (Listgarten et al. 2012; Yu et al. 2005), were used to identify associations between SNP and phenotypes. A false discovery rate method was used to reduce false positives (type I error) (Benjamini and Hochberg 1995). To test the four models, each model was evaluated using a quantile-quantile plot (testing the fit of the observed $P$ value versus the expected $P$ value of each SNP) and mean square deviation (MSD) in JMP Genomics 6.1. (Gurung et al. 2014; Kertho et al. 2015; Mamidi et al. 2011; Tamang et al. 2015).

\section{RESULTS}

Generation of genomic data sets. Genotyping of the 191 $P$. nodorum isolates was performed using the RAD-GBS technique (Baird et al. 2008) and amplification of SnToxA and SnTox3. Using RAD-GBS technique, single reads from a size-selected library of all the isolates were obtained and, in total, 4,019 $\mathrm{Mb}$ with a sequence mean read length of 148 bp were obtained from the Ion Torrent PGM sequencing platform. Isolate Nor-5 (ID: 143) was chosen as the reference genotype because it had the highest number of reads $(343,439)$. Isolates having the same nucleotide as the reference isolate at a given genomic position were scored as 0 and alternate alleles were scored as 1 . Sequence tags of Nor-5 were mapped to the reference isolate SN15 (Hane et al. 2007; Syme et al. 2013, 2016). After screening out sequence tags with low quality, with less than
$5 \%$ allele frequency, or missing data in more than $30 \%$ of the isolates, 2,983 SNP markers were identified. Genotypes of all 191 isolates with SNP were supplemented with the presence/absence polymorphism of SnToxA and SnTox3 and then used for analysis of population structure, kinship estimates, and association analyses. The average marker density was approximately one marker every $18 \mathrm{~kb}$ across the 37-Mb P. nodorum genome (Hane et al. 2007).

Population structure and kinship in the panel of the 191 isolates. Population clustering was inferred for the 191 isolates using the 2,983 markers with the software STRUCTURE. The inferred population structure identified three populations. Using a membership probability cutoff of $0.6,45$ isolates collected from North Dakota and Iran were clustered into population 1. Population 3 included 30 Australian and three South African isolates, while population 2 included a total of 78 isolates, with 63 coming from the United States, 1 from Lithuania, 5 from Switzerland, and 9 from China. The remaining 35 isolates had lower membership probabilities and were not placed into any of these three populations (Supplementary Fig. S1). The membership probability of each isolate based on $k=3$ clusters was used to form the Q matrix in additional analyses.

The pairwise kinship value was calculated based on the similarities between each pair of isolates in JMP Genomics 6.1. The average kinship value was 0.852 . The minimum kinship value of 0.8102 indicated that all 191 isolates had more than $80 \%$ similarity, with the majority having kinship values in a range between 0.8102 and 0.9709 (Supplementary Fig. S2), indicating a high degree of kinship among the 191 isolates.

LD and LD decay. Pairwise LD was calculated using all 2,983 SNP markers across all 191 isolates. The average $r^{2}$ of all the SNP pairs was 0.0432, consistent with a low LD level among the 191 isolates. LD decay was estimated by regressing the $r^{2}$ value of all SNP marker pairs on the physical distance using the nonlinear regression model. The average distance between pairwise SNP at LD $r^{2} \geq 0.8$ is $25,234 \mathrm{bp}$, indicating that, on average, SNP separated by approximately $25 \mathrm{~kb}$ will be correlated through linkage at least $80 \%$ of the time. The average distance between SNP pairs at LD $r^{2} \geq 0.9$ is 7,083 bp, indicating that, on average, SNP separated by approximately $7 \mathrm{~kb}$ are correlated through linkage at least $90 \%$ of the time. Additionally, the genome-wide LD decay defined by $r^{2}=0.1$ (Supplementary Fig. S3) was approximately $10 \mathrm{~kb}$, indicating that, on average, markers separated by more than $10 \mathrm{~kb}$ will lose association with an adjacent marker.

GWAS model evaluation and identification of marker trait association. To identify specific NE and other virulence factors important in SNB disease, four models (Q, IBS, Q+IBS, and a naïve model) were used for Alsen (harbors Tsn 1 and at least one other NE sensitivity gene) and Sumai3 (harbors Tsn1, Snn3-B1, and at least one other NE sensitivity gene) wheat. Each model was evaluated using quantile-quantile plots (Supplementary Fig. S4) and estimation of MSD (Supplementary Table S3) to identify the model with the best fit of the observed $P$ value to the expected $P$ value and lowest MSD. For both Alsen and Sumai3, the Q model and the naive model showed a better fit for the observed $P$ value to the expected $P$ value than either the IBS or Q+IBS models. The MSD of the naive and Q models also showed much lower values than either the IBS or Q+IBS models, indicating that the $Q$ and naive models performed better than the IBS and Q+IBS models.

Alsen and Sumai3 both harbor Tsn1, the host sensitivity gene corresponding to SnToxA, whereas only Sumai3 harbors Snn3-B1, the host sensitivity gene corresponding to SnTox3. An MTA between the P. nodorum NE gene SnToxA and the disease phenotype was identified in Alsen with high levels of significance $\left(-\log _{10}\right.$ $[P$ value $]=13.01$ to 17.41 ) using both the naïve and Q models (Fig. 1; Supplementary Table S4). SnToxA was also significantly associated with the disease phenotype on Sumai3 using both the naive and Q models (Fig. 1). The $-\log _{10}(P$ value) was lower than that for Alsen but still highly significant $(-\log [P$ value $]=4.61$ to 6.10$)$. SnToxA was 
not found to be significant using either the IBS or Q+IBS models. An MTA between the P. nodorum NE gene SnTox3 and the disease phenotype was identified only in Sumai3 (Fig. 1) with a high level of significance $\left(-\log _{10}[P\right.$ value $\left.]=9.10-16.85\right)$ using all four models. This was expected because Sumai3 and not Alsen harbors the SnTox3 sensitivity gene Snn3-B1.

SNPAlsen_1 and SNPAlsen_2 were identified with a high level of association with disease on Alsen $\left(-\log _{10}[P\right.$ value $]=16.71$ to 21.13) and were also highly associated with disease on Sumai3 $\left(-\log _{10}[P\right.$ value $]=6.75$ to 8.59$)$ using the naive and Q models (Fig. 1). SNP having $>30 \%$ but $<45 \%$ missing data were used to increase marker saturation and add markers that flanked the novel region. Two other SNP, SNPAlsen_3 $\left(-\log _{10}[P\right.$ value $]=8.01$ to 11.04$)$ and SNPAlsen_4 $\left(-\log _{10}[P\right.$ value $]=12.44$ to 15.72$)$, were also associated with the Alsen disease phenotypes using both the naive and Q models, and were also associated with the Sumai3 phenotypes $\left(-\log _{10}[P\right.$ value $]=5.51$ to 5.23$)$ using the naïve model (Fig. 2$)$. Given their physical proximity $(4.6 \mathrm{~kb})$, all four significant SNP (SNPAlsen_1 to SNPAlsen_4) are likely associated with the same virulence locus that interacts specifically with Alsen and Sumai3.

We investigated the genotypes of all 191 isolates for SNPAlsen_1, SNPAlsen_2, SNPAlsen_3, and SNPAlsen_4. Most of the isolates having the SNP genotype matching the Nor-5 reference sequence for SNPAlsen_1 to SNPAlsen_4 were more virulent on Alsen; likewise, isolates having the alternative SNP allele had reduced virulence on Alsen. To show the phenotypic response of spore inoculations on Alsen, eight isolates from Scandinavian populations, including four isolates (Nor-3, Nor-9, Nor-5, and Fin-5) containing the reference allele for SNPAlsen_1 to SNPAlsen_4 and four isolates (Den-1, Swe-4, Nor-6, and Nor-1) harboring the alternate alleles at these same loci, were inoculated in a new experiment (Fig. 3). An obvious correlation was found between genotype and phenotype on both Sumai3 and Alsen (Fig. 3). When averaging the phenotypes of the isolates harboring the reference allele compared with those isolates harboring the alternate allele at the two most significant SNP markers (SNPAlsen_1 and SNPAlsen_2), average disease reactions of 3.73 (range $=2.0$ to 5.0) and 2.74 (range $=0.5$ to 4.0 ) were found on Alsen for the reference and alternate alleles, respectively. Similarly, on Sumai3, average disease reactions of 3.43 (range $=0.67$ to 4.75 ) and 2.59 (range $=0.17$ to 4.75 ) were found for the reference and alternate alleles, respectively. A $t$ test showed that average disease reactions for isolates harboring the reference allele were significantly higher than for isolates harboring the alternate allele for both Alsen and Sumai3 $(P \leq 0.001)$.

Marker trait associations identified by additional GWAS analysis at the $S n T o x A$ and $S n T o x 3$ regions. Primers designed to amplify $1-\mathrm{kb}$ fragments along a $20-\mathrm{kb}$ region surrounding both SnToxA and SnTox 3 were used to identify presence/absence markers every $1 \mathrm{~kb}$. This resulted in 10 markers upstream from SnToxA and SnTox3 and 10 markers downstream. MTA were then reanalyzed using the Q model, as was done with the initial SNP marker set. Similar results came from using the naive model but the $\mathrm{Q}$ model was expected to have fewer false positives because it considers population structure; therefore, we used the Q model for analysis.

In all, 8 of the 20 markers (SnToxA_8 to SnToxA_15) were identified above the $-\log _{10}(P$ value $)$ threshold of 4.5 . Scores for the eight markers ranged from 12.63 to 17.87 for Alsen and from 5.71 to 8.93 for Sumai3 (Table 1; Fig. 4). Markers flanking the significant MTA region, including SnToxA_7 and SnToxA_16, did not reach the 4.5 significance threshold but marked an 11-kb physical region, with $5 \mathrm{~kb}$ upstream of SnToxA and $6 \mathrm{~kb}$ downstream (Fig. 4).

For the SnTox3 region, 6 of the 20 markers (SnTox3_11 to SnTox3_16) were identified above the $-\log _{10}(P$ value $)$ threshold of 4.5 and were specific to Sumai3 because Sumai3 harbors the SnTox3 sensitivity gene Snn3-B1. Scores for the six markers ranged from 17.90 to 26.26 (Table 1; Fig. 4). Markers flanking the significant MTA region, including SnTox3_10 and SnTox3_17, did not reach the 4.5 significance threshold but marked a $9-\mathrm{kb}$ physical region, with $2 \mathrm{~kb}$ upstream and $7 \mathrm{~kb}$ downstream of SnTox3 (Fig. 4).

These results indicate that the degree of recombination in the genomic region containing SnToxA was sufficient to provide highly significant MTA across an 11-kb region $(5 \mathrm{~kb}$ upstream and $6 \mathrm{~kb}$ downstream), outside of which significant MTA were not detectable. Similarly, the presence/absence markers across the genomic region containing SnTox3 provided highly significant MTA across a 9-kb region ( $2 \mathrm{~kb}$ upstream and $7 \mathrm{~kb}$ downstream), outside of which significant MTA were not detected.

Using similar methods to amplify DNA fragments across a 20-kb region surrounding the novel virulence locus containing SNPAlsen_1 to SNPAlsen_4, we found that there was little to no presence/absence or size polymorphism and, therefore, this method did not lead to additional knowledge regarding the structure of this locus. This indicates that, unlike the SnToxA and SnTox3 regions, it
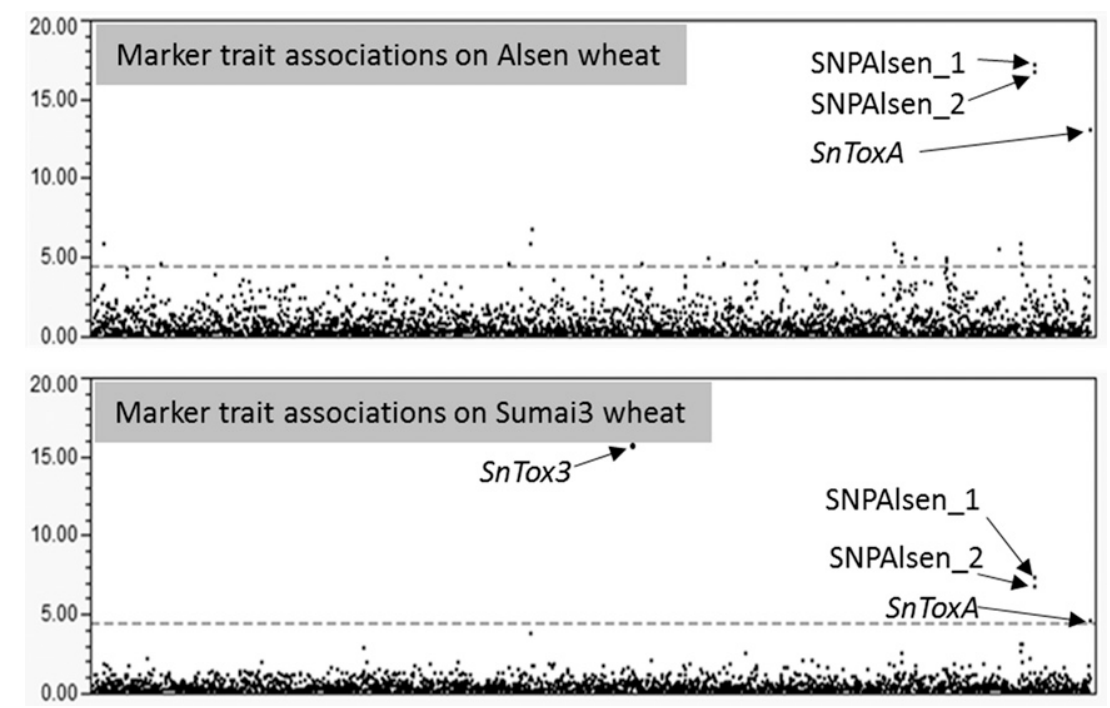

Fig. 1. Manhattan plots of marker trait association analyzed by genome-wide association study (GWAS). Single-nucleotide polymorphism (SNP) associated with Septoria nodorum blotch disease were analyzed by initial GWAS using spore inoculation data on wheat genotypes Alsen and Sumai3. The $-\log _{10}(P$ value) corrected by false discovery rate for known effector genes SnToxA and SnTox3 and the two novel SNP (SNPAlsen_1 and SNPAlsen_2) are indicated by arrows. SNP markers are represented by black dots and are ordered along the previously assembled P. nodorum contigs (Syme et al. 2016) (https://github.com/robsyme/ Parastagonospora_nodorum_SN15). A - $\log _{10}(P$ value $)$ threshold of significance of $4.5(P<0.0000316)$ appears as the gray dashed line. 
is unlikely that the differences in this novel region result from large insertions or deletions but, more likely, are due to smaller polymorphisms such as small indels or SNP that are responsible for the observed phenotypic differences. Therefore, to increase the marker set in this region, we reduced the missing data threshold from 30 to $45 \%$. This led to a total of 11 SNP (3 markers at the $30 \%$ threshold and 8 markers at the $45 \%$ threshold) across a $50-\mathrm{kb}$ region, with 4 of these SNP having significance above the $-\log _{10}$ $(P$ value) threshold of 4.5 . The closest markers flanking this region did not reach the 4.5 threshold but spanned a physical interval of $20 \mathrm{~kb}$ that provides an interval to begin evaluating candidate genes. A detailed sequence analysis of this region for all 191 isolates will be needed to identify small indels or SNP markers more tightly associated with the novel virulence phenotype.

\section{DISCUSSION}

Though P. nodorum is found in wheat fields around the world, this pathogen varies widely in the amount of damage it causes, with SNB being a major problem in some areas and a relatively minor problem in others. The differences in local disease impact reflect the effectiveness of host resistance or susceptibility in local cultivars, the virulence spectrum of the local pathogen population (McDonald et al. 2013), and local environmental factors that contribute to a compatible host-pathogen interaction. Since the first report of NE produced by $P$. nodorum (Liu et al. 2004b) and the dominant susceptibility genes that correspond to those effectors (Liu et al. 2004a), eight NE-host gene interactions that lead to NE-triggered susceptibility have been described, with three of the NE genes being cloned and functionally characterized, including SnToxA (Friesen et al. 2006), SnTox1 (Liu et al. 2012), and SnTox3 (Liu et al. 2009). Additionally, the host gene Tsnl, which confers sensitivity to SnToxA, has also been cloned and functionally characterized (Faris et al. 2010), providing a working model to characterize this interaction at the molecular level. Our working model indicates that $P$. nodorum uses NE to trigger defense response pathways, including an oxidative burst and programmed cell death to kill host cells, extract nutrients, colonize, and sporulate, resulting in the completion of its life cycle. $P$. nodorum, similar to other pathogens classified as necrotrophs, has evolved the ability to use NE to induce "host recognition" but has also evolved the ability to modulate the negative effects of the defense response that is so effective against other pathogens.

Previously, several techniques have been used to identify NE. ToxA was first identified in Pyrenophora tritici-repentis (Ballance et al. 1996; Ciuffetti et al. 1997), and we first identified SnToxA in Parastagonospora nodorum (Friesen et al. 2006) using the newly
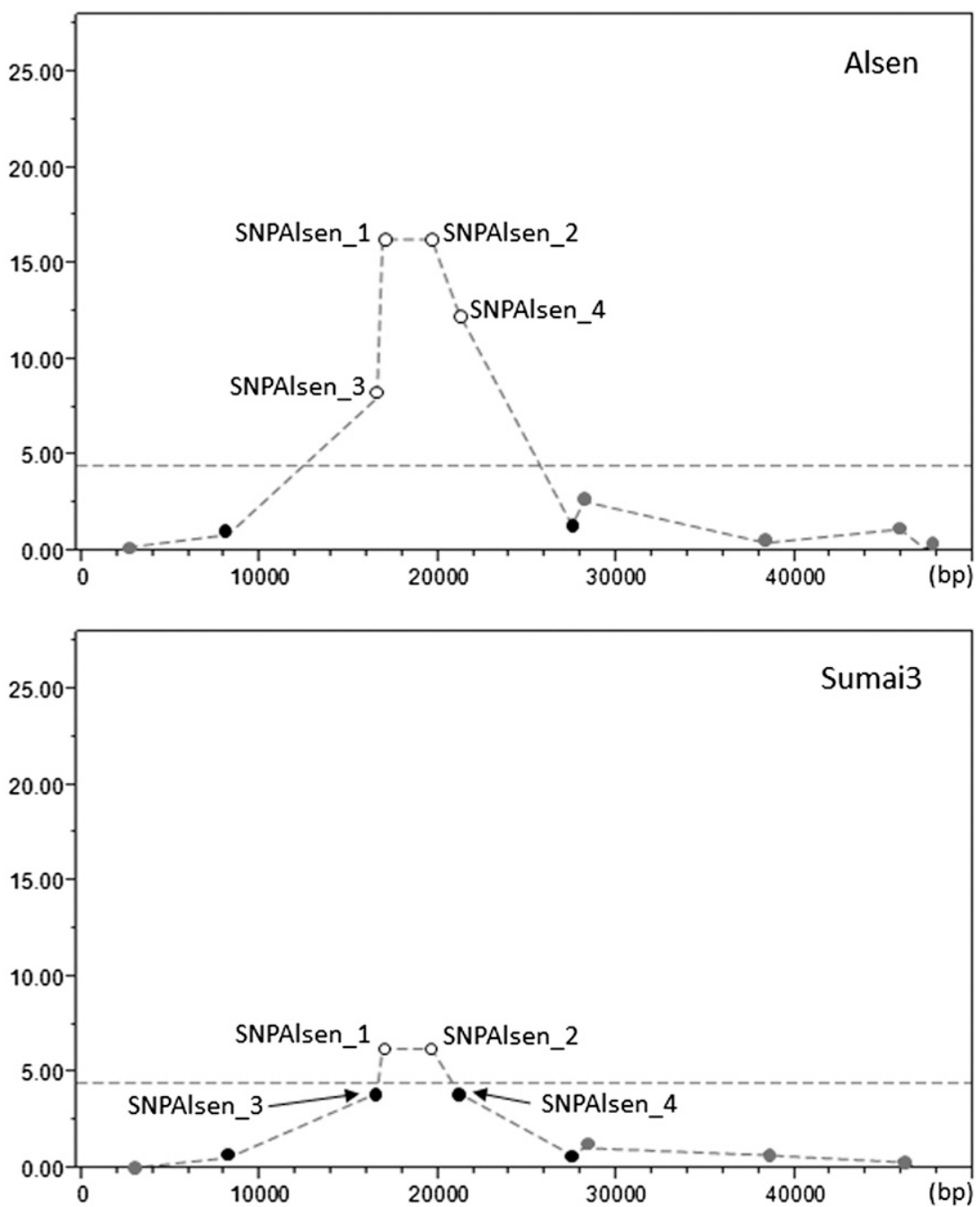

Fig. 2. Marker trait associations of markers in a 50,000-bp genomic region containing the novel locus associated with virulence phenotypes on the wheat cultivars Alsen and Sumai3. Single-nucleotide polymorphism (SNP) with significant marker trait associations (MTA) (open circles) were identified by a genome-wide association study using spore inoculation on Alsen (top) and Sumai3 (bottom). SNP including markers with a significant MTA (open circles), SNP flanking the significant markers (black circles), and other SNP without MTA significance (gray circles) are plotted according to their genomic positions ( $x$-axis) in the 50,000 -bp genomic region. Statistic probabilities of the markers are indicated by $-\log _{10}(P$ value $)$ corrected by false discovery rate $(y$-axis $)$. A $-\log _{10}(P$ value $)$ threshold of significance of $4.5(P<0.000316)$ appears as the gray dashed line. 
released $P$. nodorum genome sequence (Hane et al. 2007). We identified SnTox3 and SnTox3 using a reverse genetics approach (Liu et al. 2009) and SnToxl using a prioritized candidate gene approach (Liu et al. 2012). However, due to the quantitative nature of NE-host gene interactions and the lack of classical genetic support, the validation of novel candidate NE mentioned above is difficult and labor intensive. Several factors led us to pursue a GWAS approach in the SNB system. First and foremost, although the sexual cycle is prevalent in the field, it has been difficult to produce $P$. nodorum sexual populations in the laboratory; therefore, QTL mapping cannot be used. Fortunately, natural populations are highly recombined and composed of many genotypes and, thus, GWAS provides a powerful alternative to biparental mapping populations (Grünwald et al. in press). Additionally, once a marker set is developed in a $P$. nodorum collection, any trait that can be phenotyped can be genetically characterized. These traits can include virulence on additional host genotypes as well as traits not directly associated with virulence such as fungicide sensitivity, sensitivity to reactive oxygen, and sensitivity to pathogenesisrelated proteins. The genetic mapping resolution of GWAS has also been shown to be higher in natural populations compared with biparental populations (Leboldus et al. 2015), allowing us to quickly narrow a candidate gene region.

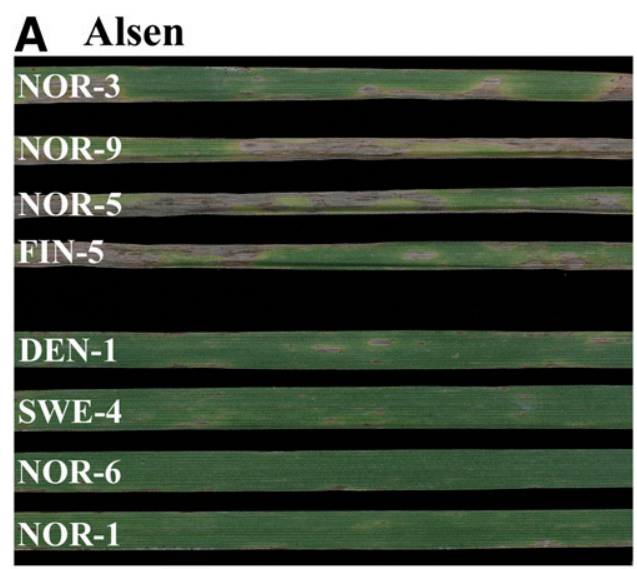

\section{B Sumai3}

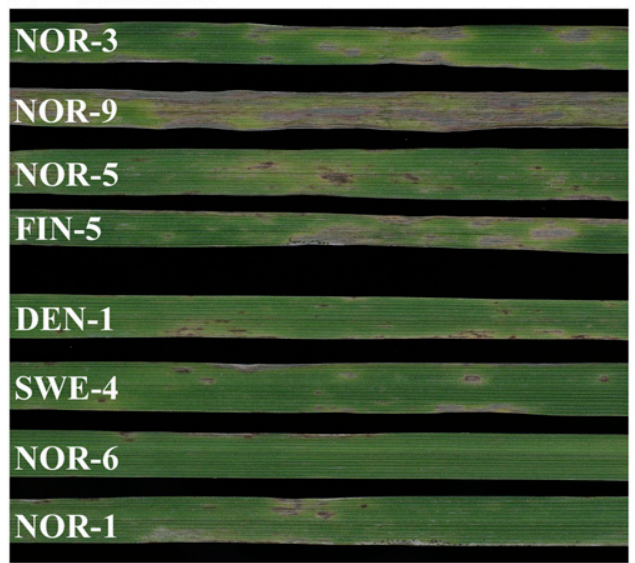

Fig. 3. Phenotypes of A, Alsen and B, Sumai3 inoculated by spores of Parastagonospora nodorum isolates harboring reference or alternative single-nucleotide polymorphism (SNP) genotypes for both SNPAlsen_1 and SNPAlsen_2. Two-week-old secondary leaves of Alsen and Sumai3 were inoculated with eight isolates from Scandinavian populations. Of the eight isolates, four isolates harbored the reference SNPAlsen_1 and SNPAlsen_2 marker alleles associated with higher virulence (Nor-3, Nor-9, Nor-5, and Fin-5) on A, Alsen (top four leaves) and B, Sumai3 (top four leaves) whereas four other Scandinavian isolates (Den-1, Swe-4, Nor-6, and Nor-1) harbored the alternate SNPAlsen_1 and SNPAlsen_2 marker alleles associated with reduced virulence on Alsen (bottom four leaves) and Sumai3 (bottom four leaves).
The success of a GWAS analysis in any organism depends on having a marker density that is greater than the degree of LD decay so as not to miss MTA for both major and minor traits. In this study, we used disease reaction type data from $191 P$. nodorum isolates collected on two popular wheat lines carrying known and unknown $\mathrm{NE}$ sensitivity genes to look for new NE or other genes associated with virulence. We included as positive controls the known NE genes SnToxA and SnTox3, which showed presence/absence polymorphism across all $191 P$. nodorum isolates, to evaluate the degree of marker density needed to identify these genes via GWAS.

Based on the degree of LD decay measured in this population, we showed that the average LD decay and, therefore, the minimum level of marker density needed to identify markers associated with a trait should be approximately one marker every $25 \mathrm{~kb}$ to identify associated genes with an $80 \%$ confidence, while markers would be required every $7 \mathrm{~kb}$ to identify associated genes with a $90 \%$ confidence. Given the genome size of $37 \mathrm{Mb}$ and an $80 \%$ confidence, we would need a minimum of approximately 1,500 markers if (i) markers were distributed evenly across all genomic regions and (ii) the rate of linkage decay was equal across the entire genome. Neither of these assumptions are likely to be correct. Using an RAD-GBS approach (Leboldus et al. 2015) to identify polymorphic SNP, we identified 2,983 high-quality SNP markers with an allele frequency $\geq 5 \%$ to be used in analyzing population structure, LD, LD decay, and MTA.

In the initial GWAS results, SnToxA and a novel virulence locus showed a highly significant association on both Alsen and Sumai3 whereas SnTox3 showed a highly significant association only on Sumai3. No other loci with high significance were identified on either Alsen or Sumai3. To evaluate the level of marker density needed to identify novel genes at any given locus, we used two methods including LD and LD decay. LD calculations showed that the average distance between marker pairs across the genome was $25,234 \mathrm{bp}$ at an $r^{2}$ value $>0.8$, which was within the range of our marker density of one marker every $18 \mathrm{~kb}$. However, LD decay analysis showed that the average marker coverage needed at $r^{2}=0.1$ was approximately $10 \mathrm{~kb}$. In a recent population genomics analysis of 213 German $F$. graminearum isolates based on 1,129 SNP markers, a similar LD decay occurred, on average, over a 1-kb interval; however, there were hotspots and coldspots of recombination across the genome and differences in rates of LD decay both among chromosomes and among field populations (Talas and McDonald 2015). In this study, the SNP density on each scaffold varied. For example, on scaffold 1, the average SNP coverage was

TABLE 1. Possibility of markers associated with traits identified by additional genome-wide association study analysis using Q model

\begin{tabular}{llcc}
\hline & & Alsen & Sumai3 \\
Marker ID & Marker type & $-\log _{10}(P$ value $)$ & $-\log _{10}(P$ value $)$ \\
\hline SnToxA & Absence/presence & 15.12 & 6.83 \\
SnToxA_8 & Absence/presence & 12.63 & 5.71 \\
SnToxA_9 & Absence/presence & 17.87 & 8.93 \\
SnToxA_10 & Absence/presence & 15.13 & 7.80 \\
SnToxA_11 & Absence/presence & 17.12 & 8.18 \\
SnToxA_12 & Absence/presence & 15.13 & 7.97 \\
SnToxA_13 & Absence/presence & 15.57 & 7.51 \\
SnToxA_14 & Absence/presence & 17.86 & 8.63 \\
SnToxA_15 & Absence/presence & 17.86 & 8.21 \\
SnTox3 & Absence/presence & Not significant & 18.05 \\
SnTox3_11 & Absence/presence & Not significant & 18.10 \\
SnTox3_12 & Absence/presence & Not significant & 20.13 \\
SnTox3_13 & Absence/presence & Not significant & 20.91 \\
SnTox3_14 & Absence/presence & Not significant & 26.26 \\
SnTox3_15 & Absence/presence & Not significant & 20.62 \\
SnTox3_16 & Absence/presence & Not significant & 17.90 \\
SNPAlsen_1 & SNP & 18.20 & 8.87 \\
SNPAlsen_2 & SNP & 18.20 & 8.86 \\
SNPAlsen_3 & SNP & 8.01 & 3.95 \\
SNPAlsen_4 & SNP & 12.44 & 3.95 \\
\hline & & &
\end{tabular}


one marker every $8 \mathrm{~kb}$ but, on scaffold 49 , the average was one SNP marker every $32 \mathrm{~kb}$. The unequal distribution may reflect the distribution of repetitive regions, which led to difficulties in identifying polymorphic markers across isolates. However, all the large scaffolds (scaffolds 1 to 45 , containing $98 \%$ of the genome) had coverage averaging at least one marker every $17 \mathrm{~kb}$. This coverage satisfies the estimated average density required (one marker approximately every $25 \mathrm{~kb}$ ) based on an LD calculation at an $r^{2}>0.8$ but not at the LD calculation at an $r^{2}>0.9$ (one marker approximately every $7 \mathrm{~kb}$ ) or an LD decay at an $r^{2}=0.1$ (one marker approximately every $10 \mathrm{~kb}$ ), indicating that more markers will be needed to confidently identify novel trait-marker associations.

Population structure was taken into account to reduce false positives in the GWAS resulting from population structure. The panel of 191 isolates was divided into three populations. Population 1 contains northern U.S. (North Dakota + Minnesota) and Iranian isolates. Population 2 contains southern, eastern, and central U.S.; Chinese; and European isolates. Population 3 contains Australian and South African isolates. The separation of these populations was based solely on the genotypes of the original 2,983 SNP. In general, population 1 and population 3 isolates were more virulent on Alsen (phenotypic scores $=3$ to 5 , except for two isolates in population 3 ), compared with population 2 isolates (phenotypic scores $=0$ to 3.5 , except for three isolates) on Alsen. The SnToxA gene region was present in all of the population 1 and most of the population 3 isolates but absent in most of the population 2 isolates. This provides evidence that selection pressure for these virulences is different in the different geographical regions, consistent with an
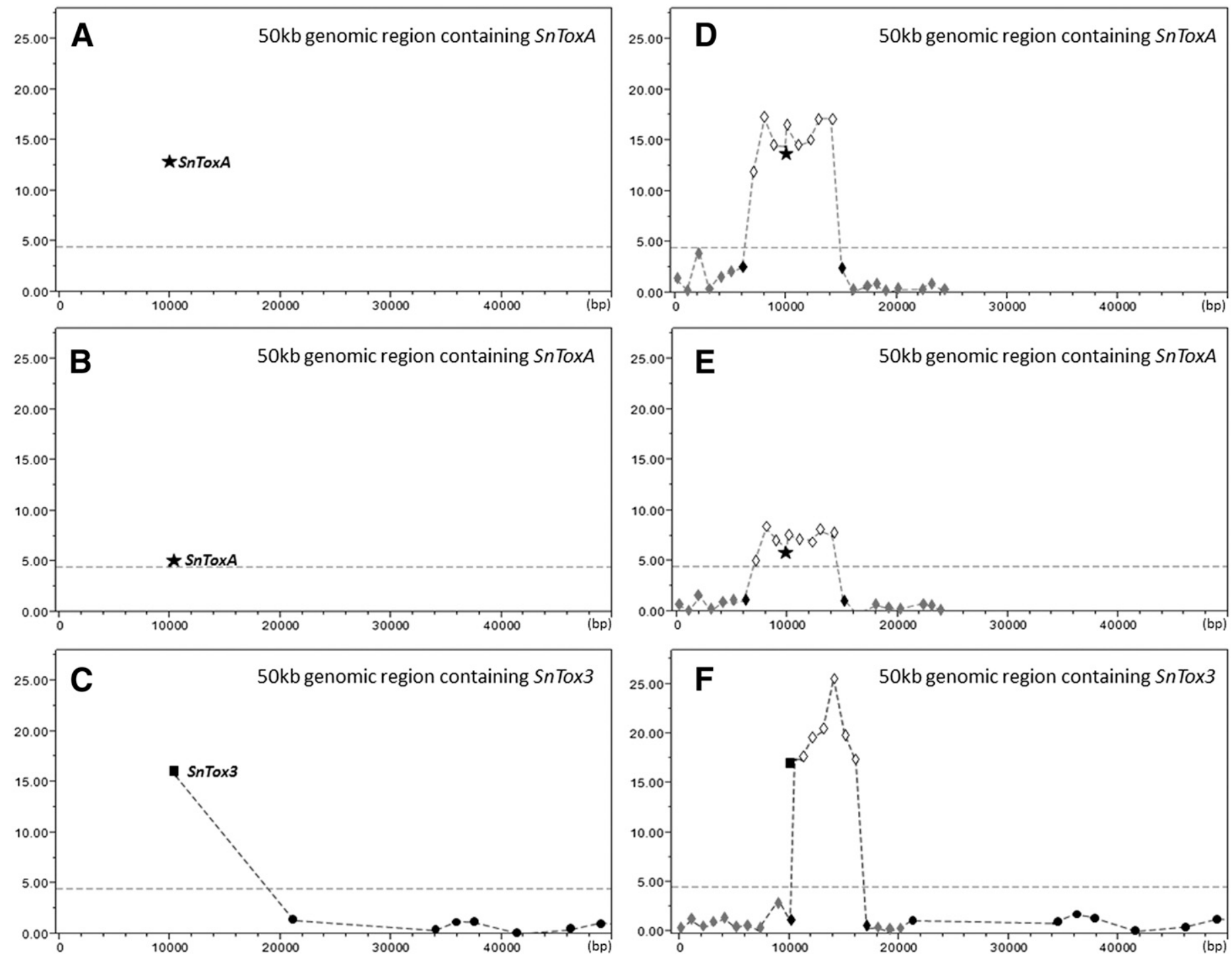

Fig. 4. Analysis of markers with significant marker trait associations (MTA) within a 50,000-bp genomic region identified by analyzing the inoculation data on the wheat genotypes Alsen and Suami3. To perform genome-wide association study analysis, presence/absence polymorphisms of necrotrophic effector genes SnToxA (black stars) and SnTox3 (black squares) were added to the marker data, including single-nucleotide polymorphism (SNP) markers (black circles) identified by the RAD-GBS method and presence/absence polymorphism markers (diamonds) generated by polymerase chain reaction amplifying every $1 \mathrm{~kb}$ along a 20 -kb region flanking SnToxA and SnTox3. Using the SNP markers and the SnToxA and SnTox3 genes as markers, SnToxA showed a significant MTA, with virulence on both A, Alsen and B, Sumai3, and SnTox3 showed a significant MTA with virulence on only C, Sumai3. By adding the presence/absence markers for the SnToxA and SnTox3 regions, an additional eight presence/absence markers (SnToxA_8 to SnToxA_15) linked with SnToxA showed significant MTA with virulence on D, Alsen and E, Suami3. An additional six presence/absence markers (SnTox3_11 to SnTox3_16) linked with SnTox3 showed significant MTA with virulence on F, Sumai3. These presence/absence markers with significant MTA are represented by open diamonds. Presence/absence markers flanking the significant markers are represented by black diamonds. Other markers without MTA significance in the 50,000-bp genomic region containing SnToxA or SnTox3 are represented by gray diamonds. All markers are plotted according to their physical genomic positions (x-axis) in the Parastagonospora nodorum contigs. Statistical probabilities of the markers are indicated by $-\log _{10}(P$ value $)$ corrected by false discovery rate $(\mathrm{y}$-axis $)$. A $-\log _{10}(P$ value $)$ threshold significance of $4.5(P<0.000316)$ appears as the gray dashed line. 
earlier analysis of effector frequencies in the global population (McDonald et al. 2013).

We were not able to identify additional presence/absence markers for the region associated with a novel virulence using a separate PCR amplification. However, four SNP markers above the $-\log _{10}$ ( $P$ values) threshold of 4.5 were identified, as well as two and five markers flanking the two sides of this region, allowing us to confidently define the interval harboring the novel gene or genes associated with virulence on both Alsen and Sumai3. It is likely that the virulence conferred by this region is not due to a large insertion or deletion as seen for SnToxA and SnTox3. We consider it likely that virulence differences in this region are due, instead, to small indels or nonsynonymous SNP within the gene or genes responsible for the phenotype. A detailed analysis of the gene sequence in this region will be required to identify candidate genes responsible for this novel virulence.

Based on our calculations of LD and LD decay, more markers will be needed to conduct future GWAS analyses in this population. However, sufficient marker density was already present to identify a novel genomic region strongly associated with virulence on both Alsen and Sumai3. Sumai3 is a Chinese wheat line that harbors good levels of resistance to Fusarium head blight (FHB), a devastating disease in many wheat-growing areas of the world. Alsen also harbors FHB resistance which, interestingly, was derived from Sumai3. It is possible that, when selecting for resistance to FHB in Alsen, the P. nodorum susceptibility was also brought in from Sumai3. In the past decade, Alsen has been planted on several million acres in the Northern Great Plains. Based on the GWAS analysis presented here, all $P$. nodorum isolates from North Dakota and Minnesota have the marker alleles associated with virulence at the SNPAlsen_1 and SNPAlsen_2 loci. However, these virulence alleles were rarely identified in U.S. isolates collected in Oregon (western United States) and the eastern part of the United States where mostly winter wheat is grown. Similar to the North Dakota/ Minnesota (north-central United States) population, the entire Australian population analyzed here also harbors the marker alleles associated with virulence at this locus. The European population, especially from the Scandinavian countries, shows segregation for this virulence (Fig. 3). Further evaluation of popular host lines in these regions will be needed to see whether they harbor the susceptibility corresponding to this virulence. We are currently evaluating host mapping populations using both Alsen and Sumai3 as parents to identify the corresponding susceptibility or resistance genes.

In this work, we used the two cloned NE genes (SnToxA and SnTox3) as positive controls to prove the utility of the GWAS approach in identifying virulence factors important in the $P$. nodorum-wheat interactions. Using these two known loci, we showed the level of marker saturation necessary to identify these virulence-associated genes. Additionally, we were able to use the SNP marker set to identify a new locus strongly associated with virulence, showing the effectiveness of this method in identifying novel genes associated with virulence. Based on the results presented here, additional marker saturation is needed to more confidently identify marker trait associations. The most efficient and cost-effective way to identify markers associated with both SNP and larger indels will be by either full-genome resequencing or deep-transcriptome sequencing. A marker set saturating the genome will facilitate the identification of genes involved in many important pathogen traits such as fungicide resistance, thermal adaptation, and novel virulence loci associated with other wheat genotypes, including both major and minor pathogen effectors involved in disease.

\section{ACKNOWLEDGMENTS}

We thank D. Holmes for technical assistance; C. Cowger, A. Ficke, J. Krupinsky, S. Ali, and P. Lipps for supplying P. nodorum isolates; M. Bolton and S. Solanki for their critical review of the manuscript; and the United States Department of Agriculture (USDA) National Institute of Food and Agriculture, Agriculture and Food Research Initiative Competitive Grant number 2016-67013-24813 for funding. Mention of trade names or commercial products in this publication is solely for the purpose of providing specific information and does not imply recommendation or endorsement by the USDA. USDA is an equal opportunity provider and employer.

\section{LITERATURE CITED}

Baird, N. A., Etter, P. D., Atwood, T. S., Currey, M. C., Shiver, A. L., Lewis, Z. A., Selker, E. U., Cresko, W. A., and Johnson, E. A. 2008. Rapid SNP discovery and genetic mapping using sequenced RAD markers. PLoS One 3:e3376.

Ballance, G. M., Lamari, L., Kowatsch, R., and Bernier, C. C. 1996. Cloning, expression and occurrence of the gene encoding the Ptr necrosis toxin from Pyrenophora tritici-repentis. Online publication. Mol. Plant. Pathol. OnLine. http://www.bspp.org.uk/mppol/1996/1209ballance/

Bastien, M., Sonah, H., and Belzile, F. 2014. Genome wide association mapping of Sclerotinia sclerotiorum resistance in soybean with a genotypingby-sequencing approach. Online publication. Plant Genome 7. doi:10.3835/ plantgenome2013.10.0030

Benjamini, Y. and Hochberg, Y. 1995. Controlling the false discovery rate: A practical and powerful approach to multiple testing. J. R. Stat. Soc. Ser. B Methodol. 57:289-300.

Bertucci, M., Brown-Guedira, G., Murphy, J. P., and Cowger, C. 2014. Genes conferring sensitivity to Stagonospora nodorum necrotrophic effectors in Stagonospora nodorum blotch-susceptible US wheat cultivars. Plant Dis. 98:746-753.

Ciuffetti, L. M., Tuori, R. P., and Gaventa, J. M. 1997. A single gene encodes a selective toxin causal to the development of tan spot of wheat. Plant Cell 9:135-144.

Connelly, C. F., and Akey, J. M. 2012. On the prospects of whole-genome association mapping in Saccharomyces cerevisiae. Genetics 191:1345-1353.

Dalman, K., Himmelstrand, K., Olson, A., Lind, M., Brandstrom-Durling, M., and Stenlid, J. 2013. A genome-wide association study identifies genomic regions for virulence in the non-model organism Heterobasidion annosum s.s. PLoS One 8:e53525.

Doerge, R. W. 2002. Mapping and analysis of quantitative trait loci in experimental populations. Nat. Rev. Genet. 3:43-52.

Earl, D. A., and vonHoldt, B. M. 2012. STRUCTURE HARVESTER: A website and program for visualizing STRUCTURE output and implementing the Evanno method. Conserv. Genet. Resour. 4:359-361.

Elshire, R. J., Glaubitz, J. C., Sun, Q., Poland, J. A., Kawamoto, K., Buckler, E. S., and Mitchell, S. E. 2011. A robust, simple genotyping-by-sequencing (GBS) approach for high diversity species. PLoS One 6:e19379.

Evanno, G., Regnaut, S., and Goudet, J. 2005. Detecting the number of clusters of individuals using the software STRUCTURE: A simulation study. Mol. Ecol. 14:2611-2620

Faris, J. D., Zhang, Z., Lu, H., Lu, S., Reddy, L., Cloutier, S., Fellers, J. P., Meinhardt, S. W., Rasmussen, J. B., Xu, S. S., Oliver, R. P., Simons, K. J., and Friesen, T. L. 2010. A unique wheat disease resistance-like gene governs effector-triggered susceptibility to necrotrophic pathogens. Proc. Natl. Acad. Sci. USA 107:13544-13549.

Friesen, T. L., and Faris, J. D. 2010. Characterization of the wheat-Stagonospora nodorum disease system: What is the molecular basis of this quantitative necrotrophic disease interaction? Can. J. Plant Pathol. 32:20-28.

Friesen, T. L., Stukenbrock, E. H., Liu, Z., Meinhardt, S., Ling, H., Faris, J. D., Rasmussen, J. B., Solomon, P. S., McDonald, B. A., and Oliver, R. P. 2006. Emergence of a new disease as a result of interspecific virulence gene transfer. Nat. Genet. 38:953-956.

Gibson, G., and Muse, S. V. 2009. Pages i to xiii in: A Primer of Genome Science, 3rd ed. Sinauer Associates, Inc., Sunderland, MA.

Grünwald, N. J., McDonald, B. A., and Milgroom, M. G. Population genomics of fungal and oomycete pathogens. Annu. Rev. Phytopathol. In press. doi: 10.1146/annurev-phyto-080614-115913

Gurung, S., Mamidi, S., Bonman, J. M., Xiong, M., Brown-Guedira, G., and Adhikari, T. B. 2014. Genome-wide association study reveals novel quantitative trait loci associated with resistance to multiple leaf spot diseases of spring wheat. PLoS One 9:e108179.

Hane, J. K., Lowe, R. G. T., Solomon, P. S., Tan, K. C., Schoch, C. L., Spatafora, J. W., Crous, P. W., Kodira, C., Birren, B. W., Galagan, J. E., Torriani, S. F. F., McDonald, B. A., and Oliver, R. P. 2007. Dothideomycete-plant interactions illuminated by genome sequencing and EST analysis of the wheat pathogen Stagonospora nodorum. Plant Cell 19:3347-3368.

Hill, W. G., and Robertson, A. 1968. Linkage disequilibrium in finite populations. Theor. Appl. Genet. 38:226-231.

Holland, J. B. 2007. Genetic architecture of complex traits in plants. Curr. Opin. Plant Biol. 10:156-161.

Kertho, A., Mamidi, S., Bonman, J. M., McClean, P. E., and Acevedo, M. 2015. Genome-wide association mapping for resistance to leaf and stripe rust in winter-habit hexaploid wheat landraces. PLoS One 10:e129580. 
Leboldus, J. M., Kinzer, K., Richards, J., Ya, Z., Yan, C., Friesen, T. L., and Brueggeman, R. 2015. Genotype-by-sequencing of the plant-pathogenic fungi Pyrenophora teres and Sphaerulina musiva utilizing Ion Torrent sequence technology. Mol. Plant Pathol. 16:623-632.

Li, H., and Durbin, R. 2010. Fast and accurate long-read alignment with Burrows-Wheeler transform. Bioinformatics 26:589-595.

Listgarten, J., Lippert, C., Kadie, C. M., Davidson, R. I., Eskin, E., and Heckerman, D. 2012. Improved linear mixed models for genome-wide association studies. Nat. Methods 9:525-526.

Liu, Z., Faris, J., Meinhardt, S., Ali, S., Rasmussen, J., and Friesen, T. 2004a. Genetic and physical mapping of a gene conditioning sensitivity in wheat to a partially purified host-selective toxin produced by Stagonospora nodorum. Phytopathology 94:1056-1060.

Liu, Z., Faris, J. D., Oliver, R. P., Tan, K. C., Solomon, P. S., McDonald, M. C., McDonald, B. A., Nunez, A., Lu, S., and Rasmussen, J. B. 2009. SnTox3 acts in effector triggered susceptibility to induce disease on wheat carrying the Snn3 gene. PLoS Pathog. 5:e1000581.

Liu, Z., Friesen, T., Rasmussen, J., Ali, S., Meinhardt, S., and Faris, J. 2004b. Quantitative trait loci analysis and mapping of seedling resistance to Stagonospora nodorum leaf blotch in wheat. Phytopathology 94:1061-1067.

Liu, Z., Zhang, Z., Faris, J. D., Oliver, R. P., Syme, R., McDonald, M. C., McDonald, B. A., Solomon, P. S., Lu, S., Shelver, W. L., and Xu, S. 2012. The cysteine rich necrotrophic effector SnTox1 produced by Stagonospora nodorum triggers susceptibility of wheat lines harboring Snn1. PLoS Pathog. 8:e1002467.

Mamidi, S., Chikara, S., Goos, R. J., Hyten, D. L., Annam, D., Moghaddam, S. M., Lee, R. K., Cregan, P. B., and McClean, P. E. 2011. Genome-wide association analysis identifies candidate genes associated with iron deficiency chlorosis in soybean. Plant Genome 4:154-164.

McDonald, M. C., Oliver, R. P., Friesen, T. L., Brunner, P. C., and McDonald, B. A. 2013. Global diversity and distribution of three necrotrophic effectors in Phaeosphaeria nodorum and related species. New Phytol. 199:241-251.

Montgomery, G. W. 2011. Genome-wide association studies and genetic architecture of common human diseases. BMC Proc. 5:S16.

Muller, L. A. H., Lucas, J. E., Georgianna, D. R., and McCusker, J. H. 2011. Genome-wide association analysis of clinical vs. nonclinical origin provides insights into Saccharomyces cerevisiae pathogenesis. Mol. Ecol. 20: 4085-4097.

Nordborg, M., and Tavare, S. 2002. Linkage disequilibrium: What history has to tell us. Trends Genet. 18:83-90.

Palaisa, K., Morgante, M., Tingey, S., and Rafalski, A. 2004. Long-range patterns of diversity and linkage disequilibrium surrounding the maize $Y 1$ gene are indicative of an asymmetric selective sweep. Proc. Natl. Acad. Sci. USA 101:9885-9890.

Palma-Guerrero, J., Hall, C. R., Kowbel, D., Welch, J., Taylor, J. W., Brem, R. B., and Glass, N. L. 2013. Genome wide association identifies novel loci involved in fungal communication. PLoS Genet. 9:e1003669.
Pasam, R. K., Sharma, R., Malosetti, M., van Eeuwijk, F. A., Haseneyer, G., Kilian, B., and Graner, A. 2012. Genome-wide association studies for agronomical traits in a world wide spring barley collection. BMC Plant Biol. 12:16.

Pritchard, J. K., Stephens, M., and Donnelly, P. 2000. Inference of population structure using multilocus genotype data. Genetics 155:945-959.

Remington, D. L., Thornsberry, J. M., Matsuoka, Y., Wilson, L. M., Whitt, S. R., Doebley, J., Kresovich, S., Goodman, M. M., and Buckler, E. S. 2001. Structure of linkage disequilibrium and phenotypic associations in the maize genome. Proc. Natl. Acad. Sci. USA 98:11479-11484.

Shi, G., Zhang, Z., Friesen, T. L., Bansal, U., Cloutier, S., Wicker, T., Rasmussen, J. B., and Faris, J. D. 2016. Marker development, saturation mapping, and high-resolution mapping of the Septoria nodorum blotch susceptibility gene Snn3-B1 in wheat. Mol. Genet. Genomics 291:107-119.

Stevens, E. L., Heckenberg, G., Roberson, E. D. O., Baugher, J. D., Downey, T. J., and Pevsner, J. 2011. Inference of Relationships in population data using identity-by-descent and identity-by-state. PLoS Genet. 7:e1002287.

Syme, R. A., Hane, J. K., Friesen, T. L., and Oliver, R. P. 2013. Resequencing and comparative genomics of Stagonospora nodorum: Sectional gene absence and effector discovery. G3: Genes Genomes Genet. 3:959-969.

Syme, R. A., Tan, K. C., Hane, J. K., Dodhia, K., Stoll, T., Hastie, M., Furuki, E., Ellwood, S. R., Williams, A. H., Tan, Y. F., and Testa, A. C. 2016. Comprehensive annotation of the Parastagonospora nodorum reference genome using next-generation genomics, transcriptomics and proteogenomics. PLoS One 11:e0147221.

Talas, F., Kalih, R., Miedaner, T., and McDonald, B. A. 2016. Genome-wide association study identifies novel candidate genes for aggressiveness, deoxynivalenol production and azole sensitivity in natural field populations of Fusarium graminearum. Mol. Plant-Microbe Interact. 29:417-430.

Talas, F., and McDonald, B. A. 2015. Genome-wide analysis of Fusarium graminearum field populations reveals hotspots of recombination. BMC Genomics 16:996.

Tamang, P., Neupane, A., Mamidi, S., Friesen, T., and Brueggeman, R. 2015. Association mapping of seedling resistance to spot form net blotch in a worldwide collection of barley. Phytopathology 105:500-508.

Yu, J. M., Pressoir, G., Briggs, W. H., Bi, I. V., Yamasaki, M., Doebley, J. F., McMullen, M. D., Gaut, B. S., Nielsen, D. M., Holland, J. B., Kresovich, S., and Buckler, E. S. 2005. A unified mixed-model method for association mapping that accounts for multiple levels of relatedness. Nat. Genet. 38: 203-208.

Zhao, Y., Wang, H., Chen, W., and Li, Y. 2014. Genetic structure, linkage disequilibrium and association mapping of Verticillium wilt resistance in elite cotton (Gossypium hirsutum L.) germplasm population. PLoS One 9:e86308.

Zhu, C., Gore, M., Buckler, E. S., and Yu, J. 2008. Status and prospects of association mapping in plants. Plant Genome 1:5-20. 